Çukurova Üniversitesi Mühendislik Fakültesi Dergisi, 36(3), ss. 755-767, Eylül 2021

Çukurova University Journal of the Faculty of Engineering, 36(3), pp. 755-767, September 2021

\title{
Yapısal Olarak Değiştirilmiş HMX-DFT Çalışması
}

\author{
Mehmet Erman MERT ${ }^{* 1}$ \\ ${ }^{1}$ Adana Alparslan Türkeş Bilim ve Teknoloji Üniversitesi, Illeri Teknolojiler Uygulama ve \\ Araştırma Merkezi, Adana
}

Geliş tarihi: 05.04.2021

Kabul tarihi: 13.09.2021

\section{Öz}

Oktahidro-1,3,5,7-tetranitro-1,3,5,7-tetrazosin (HMX) askeri ve endüstriyel uygulamalarda yaygın olarak kullanılan nitramin türevi bir patlayıcıdır. HMX ve benzeri özelliklere sahip patlayıcılar için duyarlılık önemli bir parametredir. Duyarlılığın düşürülmesi sayesinde 1sı, sıcaklık, çarpma, sürtünme ile statik elektrik deşarjı gibi çeşitli olayların oluşmasıyla meydana gelebilecek kontrolsüz patlamalar önlenebilir. $\mathrm{Bu}$ amaçla, çeşitli katkı maddeleri kullanmak veya patlayıcı etkide önemli bir kayıba neden olmaksızın kimyasal modifikasyonlar yapılmaktadır. $\mathrm{HMX}\left(\mathrm{C}_{4} \mathrm{H}_{8} \mathrm{~N}_{8} \mathrm{O}_{8}\right)$ molekülünün 4 tane nitro grubu vardır. $\mathrm{Bu}$ çalışmada, nitro gruplarının nitrozo ve amino gruplarına dönüştürülmesiyle duyarlılığı düşürmenin mümkün olabileceği düşünülmektedir. Balistik özellikler, yani patlama hızı (D) ve patlama basıncı (P) Kamlet-Jacobs denklemleri kullanılarak incelenmiştir. Elde edilen sonuçlara göre, belirtilen moleküler modifikasyonlarla, HMX'in duyarlılığının düşürülebileceği tespit edilmiştir.

Anahtar Kelimeler: HMX (Oktojen), Patlama basıncı, Patlama hızı, Yoğunluk fonksiyonu teorisi

\section{Structurally Modified HMX-A DFT Study}

\begin{abstract}
Octahydro-1,3,5,7-tetranitro-1,3,5,7-tetrazosine (HMX) is a nitramine explosive derivative that widely used in military and industrial applications. The sensitivity is a significant parameter for HMX and explosives which have similar properties. By reducing the sensitivity, uncontrolled explosions that may occur due to various events such as heat, temperature, impact, friction and static electricity discharge can be prevented. For this purpose, chemical modifications are made without using various additives or causing a significant loss in explosive effect. $\mathrm{HMX}\left(\mathrm{C}_{4} \mathrm{H}_{8} \mathrm{~N}_{8} \mathrm{O}_{8}\right)$ molecule has 4 nitro groups. In this study, it is thought that it may be possible to reduce the sensitivity by converting these nitro groups into nitroso and amino groups. Ballistic properties, ie detonation velocity (D) and detonation pressure (P), are investigated using Kamlet-Jacobs equations. According to the results, it was determined that the sensitivity of HMX can be decreased with the specified molecular modifications.
\end{abstract}

Keywords: HMX (Octogen), Detonation pressure, Detonation velocity, Density function theory

*Sorumlu yazar (Corresponding author): Mehmet Erman MERT, memert@atu.edu.tr 


\section{GíRiş}

Günümüzde enerjik malzemeler üzerine yapılan teorik çalışmalar moleküler modellemenin gelişimi ile günden güne hız kazanmıştır. Geliştirilen moleküler modelleme programları sayesinde moleküllerin geometrileri, enerjileri, hal değişimleri, kimyasal etkinlikleri, IR, UV ve NMR spektrumları, bir enzim ile bir yüzeyin etkileşimi ve maddelerin fiziksel özellikleri gibi bir takım özellikler hakkında bilgi sahibi olunmaktadır. Bu sayede yapılacak olası deneysel çalışmalar hakkında önceden bilgi sahibi olunabilmekte ve mevcut çalışmalar ise geliştirilebilmektedir [1-3].

Oktahidro -1,3,5,7- tetranitro -1,3,5,7- tetrazosin (HMX), patlay1c1 bir polinitramindir [4]. Kimyasal formülü C4H8N8O8 olan ve yaklaşık $286^{\circ} \mathrm{C}$ erime noktasına sahip renksiz bir katıdır [5]. HMX, hekzaminin asetik asit/asetik anhidrit çözücüsü içinde amonyum nitrat ve nitrik asit ile nitratlanmasiyla sentezlenir [6]. Yap1 olarak HMX'e benzer bir başka patlayıcı olan siklotrimetilen-trinitramin (RDX) sentezi sırasında da az miktarda HMX oluşur. Termal kararlılı̆̆ı ve sahip olduğu yüksek balistik özellikleri nedeniyle diğer patlayıcılara göre askeri ve endüstriyel uygulamalar için önemli bir malzeme haline gelmiştir [7,8]. HMX, yüksek sıcaklıklarda $\left(279^{\circ} \mathrm{C}\right.$ ve üzeri) şiddetli bir şekilde patlar [5]. Bu özellik nedeniyle, nükleer cihazlarda parçalanabilir malzemeyi patlatmak için, plastik bağlı patlayıcıların bir bileşeni olarak, roket itici yakıtının bir bileşeni olarak sıklıkla kullanılmaktadır [9].

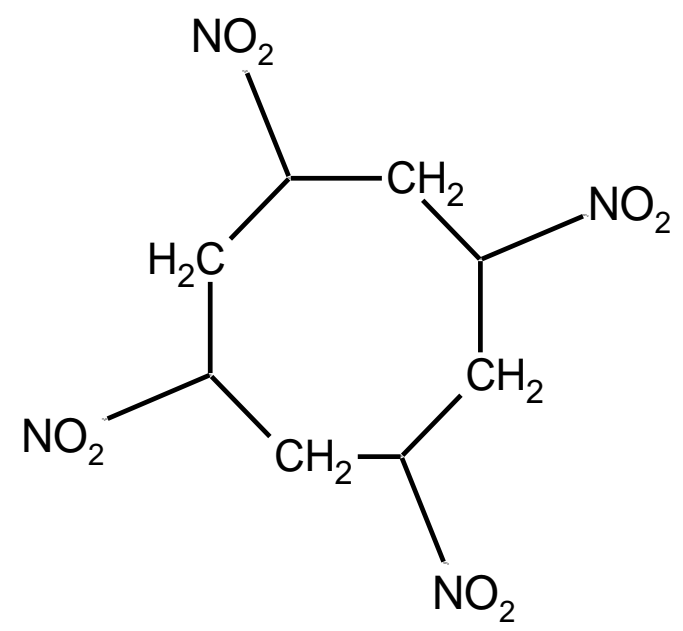

Şekil 1. HMX molekülü

Patlayıc1 hassasiyetinin azaltılması ile ilgili literatürde birçok çalışma bulunmaktadır [10-15]. $\mathrm{Bu}$ kapsamda gerçekleştirilen yöntemlerden ilki katkı maddelerinin ve patlayıcı kaplamalarının kullanılmasıdır. İkinci yöntem ise bu çalışmada da sunulduğu gibi, patlayıcı molekülün yapısında balistik özelliklerini fazla değiştirmeden moleküler düzeyde değişiklikler yapmaktır. Şekil 1'den görüleceği üzere HMX molekülü 4 tane nitro grubu içermektedir. $\mathrm{Bu}$ nitro $\left(-\mathrm{NO}_{2}\right)$ gruplarının nitrozo (-NO) ve amino $\left(-\mathrm{NH}_{2}\right)$ gruplarına dönüştürülmesinin (Şekil 2) duyarlılığ1 azaltabileceği varsayılmaktadır. HMX'ten türetilen moleküllerin balistik özelliklerinin anlaşılması, yapısal modifikasyon yoluyla patlayıcıların duyarlılığını değiştirmede kullanılabilecek faktörleri ortaya koymaktadır. Duyarsızlaştırma sayesinde türetilen moleküller, başlangıç molekülüne kıyasla çok daha güvenli patlayıcılar olabilir ve 1sı, sıcaklık, çarpma, sürtünme ile statik elektrik deşarjı gibi çeşitli olayların oluşması ile meydana gelen kontrolsüz patlamaları önleyebilir. 


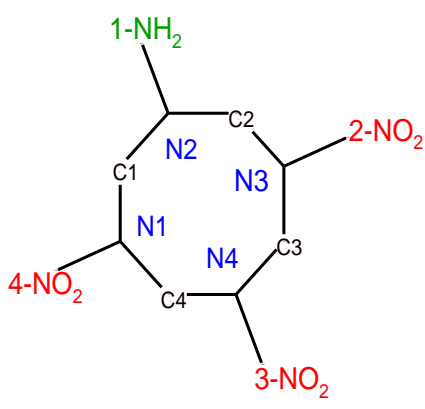

1-(1) $\mathrm{NH}_{2}$

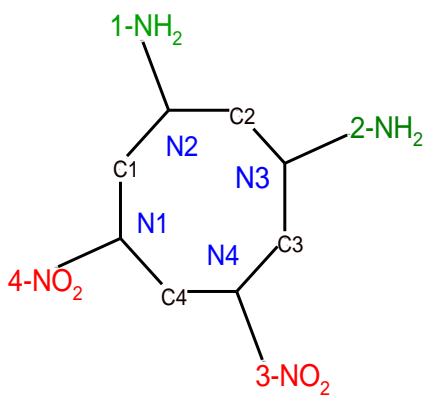

2-(2) $\mathrm{NH}_{2}-1$

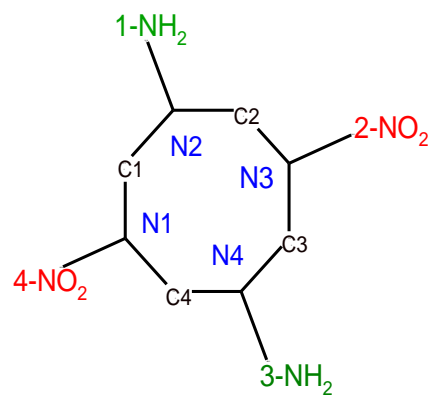

3-(2) $\mathrm{NH}_{2}-2$

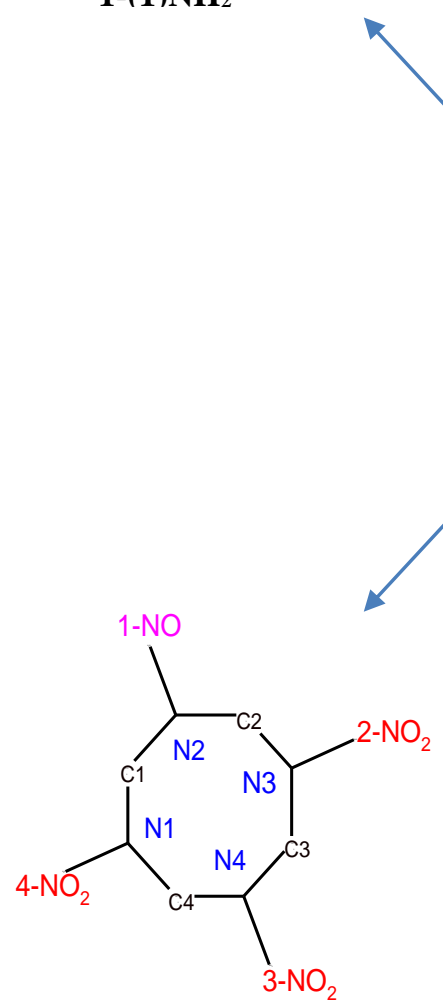

4-(1)NO
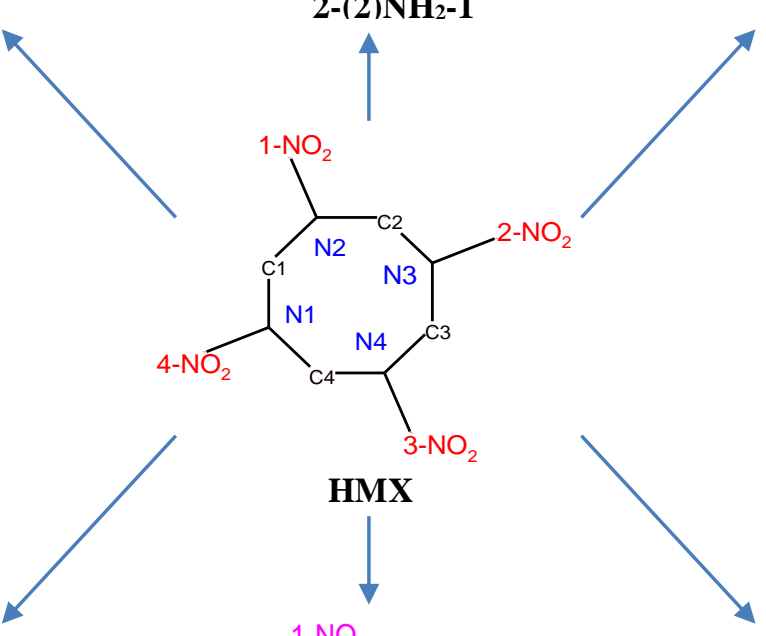

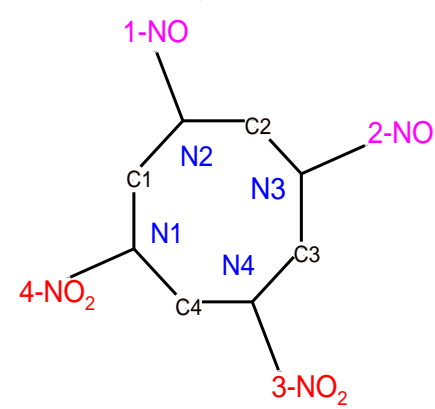

5-(2)NO-1

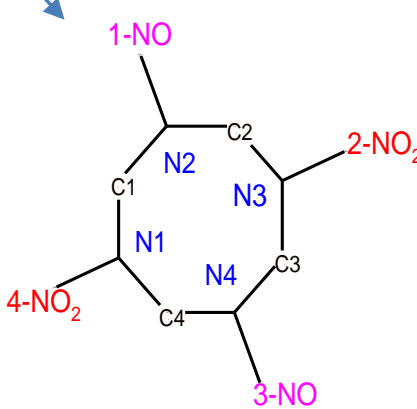

6-(2)NO-2

Şekil 2. HMX ve ondan üretilen moleküllerin şekilleri

\section{MATERYAL VE METOT}

Çalışmada incelenen moleküller için yapılan teorik hesaplamalar Spartan 14 ve Gaussian 03Wpaket programlar1 ile B3LYP/6-31G** (d,p) temel setinde yapılmıştır [16,17].
$\mathrm{Bu}$ çalışmada, Spartan 14 paket programında HMX molekülünün üç boyutlu yapısı çizilerek, programın "calculations" ara yüzü açıldı. "calculate" bölümüne "Equilibrium Geometry" komutu, "compute" kismina "IR" ve "Print" kısmına ise "Thermodynamics" komutları girildi. Temel durum ve geçiş durumu geometrileri ve 
termodinamik özellikler gibi birçok amaç için güvenilir sonuçlar ortaya koyan [18-21] HartreeFock yöntemiyle vakumda moleküle sirasiyla STO-3G, 3-21G, 6-31G* ve 6-31G** temel setleri uygulandı. Akabinde HMX molekülüne Yoğunluk Fonksiyonu Teorisi DFT/B3LYP düzeyinde sirasiyla $6-31 \mathrm{G}^{*}$ ve $6-31 \mathrm{G}^{* *}$ temel setleri uygulanarak molekülün geometrik optimizasyonu yapıldı. Bu sayede elde edilen yapı Şekil 3'de verilmiştir.

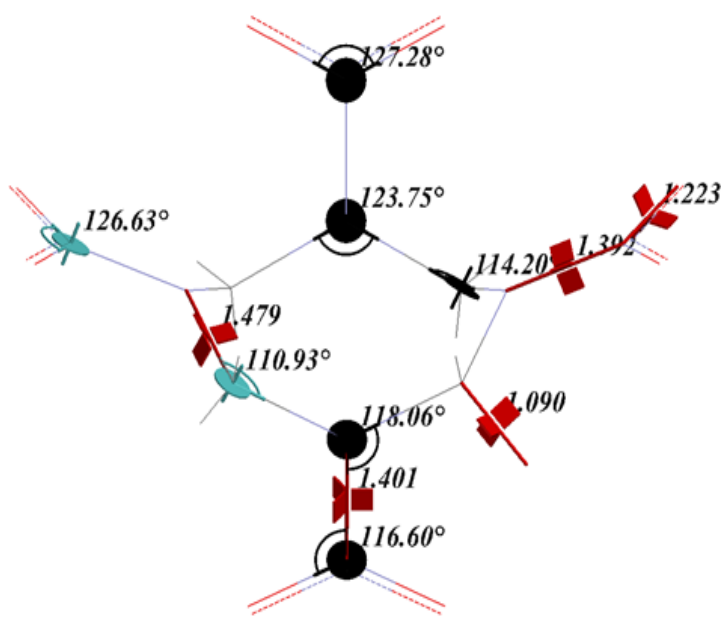

Şekil 3. Optimize edilmiş HMX molekülü

DFT/B3LYP düzeyinde 6-31G** temel sette HMX molekülünün enerji hesabı yapılarak çeşitli termodinamik özellikleri hesaplandı. Ardından, HMX molekülü için Spartan 14 programında Parametrizasyon metodu (PM3) kullanılarak, molekülün standart oluşum entalpi $\left(\Delta \mathrm{H}_{\mathrm{f}}^{\circ}\right)$ değeri hesapland1.

Enerjik bileşiklerin balistik niteliklerini etkileyen özelliklerden biri olan yoğunluğu hesaplamak için en yaygın kullanılan yöntemlerden birisi Monte Carlo metodudur [8,23-25]. Bu nedenle optimize edilen HMX molekülünün teorik yoğunluk hesabını yapmak için Gaussian 03W paket programında yer alan Monte Carlo metodu kullanılarak molekülün molar hacmi 100 defa hesaplanmış ve aritmetik ortalaması alınmıştır. Molekülün mol kütlesinin bulunan ortalama molar hacmine bölünmesiyle HMX molekülü için teorik yoğunluk değeri hesaplanmıştır.
$\mathrm{C}_{\mathrm{a}} \mathrm{H}_{\mathrm{b}} \mathrm{O}_{\mathrm{c}} \mathrm{N}_{\mathrm{d}}$ molekül yapısına sahip patlayıcı molekülleri için kullanılan stokiyometrik ilişki $(2 \mathrm{a}+\mathrm{b} / 2>\mathrm{c} \geq \mathrm{b} / 2$ ise $\mathrm{N}=(\mathrm{b}+2 \mathrm{c}+2 \mathrm{~d}) / 4 \mathrm{M}$, $\mathrm{M}=(56 \mathrm{~d}+88 \mathrm{c}-8 \mathrm{~b}) /(\mathrm{b}+2 \mathrm{c}+2 \mathrm{~d}), \quad \mathrm{Q}=[28.9 \mathrm{~b}+94.05$ $\left.(\mathrm{c} / 2-\mathrm{b} / 4)+0.239 \quad \Delta \mathrm{H}_{\mathrm{f}}^{\mathrm{o}} \mathrm{j} / \mathrm{M}\right) \quad[26] \quad$ kullanılarak molekülün $\mathrm{N}, \mathrm{M}$ ve $\mathrm{Q}$ değerleri hesaplanmıştır [27].

Eşitlik 1 ve 2'de verilen Kamlet-Jacobs denklemleri [26,28-30] kullanılarak HMX molekülünün teorik olarak patlama hızı (D) ve patlama basıncı $(\mathrm{P})$ hesaplanmıştır.

$\mathrm{D}=1,01\left(\mathrm{~N} \mathrm{M}^{1 / 2} \mathrm{Q}^{1 / 2}\right)^{1 / 2}(1+1,30 \rho)$

$\mathrm{P}=1,558 \rho^{2} \mathrm{NM}^{1 / 2} \mathrm{Q}^{1 / 2}$

D: Patlama hızı (km/s), P: Patlama basıncı (GPa), $\rho$ : bileşiğin yoğunluğu $\left(\mathrm{g} / \mathrm{cm}^{3}\right), \mathrm{N}$ : patlayıcının gramı başına düşen patlama ürünlerinden oluşan gazın molü $(\mathrm{mol} / \mathrm{g}), \mathrm{M}_{\text {ort. }}$ : oluşan gaz ürünlerinin ortalama mol kütlesi $(\mathrm{g} / \mathrm{mol})$, Q: patlamanın kimyasal enerjisi $(\mathrm{kJ} / \mathrm{g})$.

Eşitlik 3 kullanılarak ise oksijen içeriği hesaplanmıştır [20].

$\% \Omega=\frac{\mu_{0}}{\mu_{\mathrm{pat}}}\left(\mathrm{c}-2 \mathrm{a}-\frac{\mathrm{b}}{2}\right) \times 100$

Patlama reaksiyonunun bir sonucu olarak ortaya çıkabilecek olası gaz ürünleri, KistiakowskyWilson kuralları [22]:

1- Oksijen atomları, ' $\mathrm{H}$ ' atomlarını ' $\mathrm{H}_{2} \mathrm{O}$ ' moleküllerine okside eder. $\left(2 \mathrm{H}+\mathrm{O} \rightarrow \mathrm{H}_{2} \mathrm{O}_{(\mathrm{g})}\right)$

2- Kalan ' $\mathrm{O}$ ' atomları, tüm ' $\mathrm{C}$ ' atomlarını $\mathrm{CO}$ moleküllerine oksitler. $\left(\mathrm{C}+\mathrm{O} \rightarrow \mathrm{CO}_{(\mathrm{g})}\right)$

3- Kalan 'O' atomları, tüm 'CO' moleküllerini 'CO2' moleküllerine okside eder. $\left(\mathrm{CO}+\mathrm{O} \rightarrow \mathrm{CO}_{2(\mathrm{~g})}\right)$

4- $\mathrm{O}, \mathrm{H}$ ve $\mathrm{N}$ atomlarının fazlası $\mathrm{O}_{2}, \mathrm{H}_{2}$ ve $\mathrm{N}_{2}$ 'ye dönüştürülür. Fazla $\mathrm{C}, \mathrm{C}$ (katı) olarak kalır, $\left(2 \mathrm{H} \rightarrow \mathrm{H}_{2(\mathrm{~g})}, \quad 2 \mathrm{~N} \rightarrow \mathrm{N}_{2(\mathrm{~g})}, \quad 2 \mathrm{O} \rightarrow \mathrm{O}_{2(\mathrm{~g})}\right)$ yardımıyla belirlenmiştir. 
HMX molekülü için elde edilen teorik değerlerle literatür değerleri karşılaştırılmıştır. Tasarlanan yeni moleküller içinde yukarıda sunulan işlemler sırasıyla uygulanmıştır.

\section{ARAŞTIRMA BULGULARI}

\subsection{Geometriler}

HMX ve ondan türetilen moleküllerin optimize edilmiş yapıları Şekil 4'de görülmektedir. Teorik olarak hesaplanmış bağ açıları ve bağ uzunlukları ise sırasıyla Çizelge 1 ve Çizelge 2'de verilmiştir. HMX molekülü için literatürden elde edilen değerler ile belirlenen veriler arasındaki ilişki değerlendirildiğinde parelel sonuçların elde edildiği görülmektedir. Bu durum, moleküllerin geometri optimizasyonunun başarılı bir şekilde gerçekleştirildiğini göstermektedir. HMX molekülündeki $\mathrm{NO}_{2}$ grubunun $\mathrm{NO}$ veya $\mathrm{NH}_{2}$ ile yer değiştirmesi sonucu bağ uzunluklarının değiştiği görülmektedir. $\mathrm{NH}_{2}$ ile yerdeğiştirdiğinde bağ uzunluğunun arttığı, NO eklenmeside ise azaldığ1 görülebilmektedir.

Çizelge 1. HMX molekülü için bu çalışmada elde edilen ve literatür verilerinden elde edilen bağ açıları $\left(\left(^{\circ}\right)\right.$

\begin{tabular}{|c|c|c|c|c|c|c|}
\hline Bă Açıları & HMX & {$[\mathbf{3 1}]$} & {$[\mathbf{3 2}]$} & {$[\mathbf{3 3}]$} & {$[\mathbf{3 4}]$} & {$[\mathbf{3 5}]$} \\
\hline N-N-O & $116,36-118,69$ & 116,8 & 117 & 116,56 & $116,6-119,9$ & 117,28 \\
\hline O-N-O & $126,63-127,28$ & 126,3 & 127 & 127,10 & $123,9-128,2$ & 125,65 \\
\hline C-N-N & $116,72-113,58$ & 117,3 & 117 & & $115,9-114,3$ & 119,94 \\
\hline C-N-C & $123,64-123,75$ & 123,1 & 122 & 123,15 & $124,3-125,3$ & \\
\hline N-C-H & $109,45-109,05$ & & & & & 109,86 \\
\hline N-C-N & $114,20-110,93$ & 111,8 & 114 & 111,23 & $114,4-108,7$ & \\
\hline
\end{tabular}

Çizelge 2. HMX ve ondan türetilen moleküller için elde edilen bağ uzunlukları( $)$ a:[36], b: [37], c: [33]

\begin{tabular}{|c|c|c|c|c|c|c|c|c|}
\hline Bağ Uzunluğu & HMX & Literatür & $\mathbf{1}$ & $\mathbf{2}$ & $\mathbf{3}$ & $\mathbf{4}$ & $\mathbf{5}$ & $\mathbf{6}$ \\
\hline $\mathrm{N} 1-\mathrm{C} 1$ & 1,450 & $1,450^{\mathrm{b}}$ & 1,480 & 1,500 & 1,466 & 1,453 & 1,454 & 1,453 \\
\hline $\mathrm{C} 1-\mathrm{N} 2$ & 1,456 & $1,450^{\mathrm{a}}$ & 1,439 & 1,454 & 1,479 & 1,456 & 1,447 & 1,448 \\
\hline $\mathrm{N} 2-\mathrm{C} 2$ & 1,456 & $1,458^{\mathrm{a}}$ & 1,438 & 1,466 & 1,473 & 1,451 & 1,454 & 1,456 \\
\hline $\mathrm{C} 2-\mathrm{N} 3$ & 1,450 & $1,447^{\mathrm{a}}$ & 1,486 & 1,489 & 1,473 & 1,453 & 1,458 & 1,452 \\
\hline $\mathrm{N} 3-\mathrm{C} 3$ & 1,450 & $1,451^{\mathrm{c}}$ & 1,449 & 1,479 & 1,495 & 1,450 & 1,451 & 1,452 \\
\hline $\mathrm{C} 3-\mathrm{N} 4$ & 1,456 & $1,456^{\mathrm{a}}$ & 1,456 & 1,467 & 1,466 & 1,456 & 1,454 & 1,456 \\
\hline $\mathrm{N} 4-\mathrm{C} 4$ & 1,456 & $1,453^{\mathrm{c}}$ & 1,460 & 1,468 & 1,454 & 1,453 & 1,450 & 1,448 \\
\hline $\mathrm{C} 4-\mathrm{N} 1$ & 1,450 & $1,452^{\mathrm{c}}$ & 1,446 & 1,469 & 1,501 & 1,453 & 1,455 & 1,453 \\
\hline $\mathrm{N} 1-4-\mathrm{NO} 2$ & 1,404 & $1,405^{\mathrm{a}}$ & 1,399 & 1,453 & 1,436 & 1,413 & 1,401 & 1,402 \\
\hline $\mathrm{N} 2-1-\mathrm{NO} 2$ & 1,416 & $1,413^{\mathrm{b}}$ & 1,421 & 1,442 & 1,507 & 1,377 & 1,378 & 1,381 \\
\hline $\mathrm{N} 3-2-\mathrm{NO} 2$ & 1,404 & $1,398^{\mathrm{c}}$ & 1,388 & 1,511 & 1,434 & 1,411 & 1,375 & 1,402 \\
\hline $\mathrm{N} 4-3-\mathrm{NO} 2$ & 1,416 & $1,416^{\mathrm{a}}$ & 1,410 & 1,432 & 1,431 & 1,402 & 1,410 & 1,381 \\
\hline
\end{tabular}

\subsection{Elektrostatik Potansiyel}

Elektrostatik potansiyellerin belirlenmesi sayesinde, molekülün üç boyutlu olarak yük dağılımları, kimyasal tepkimelere girme eğilimi, hidrojen bağı etkileşimleri, elektronca zengin yani pozitif yüklü (elektrofilik) bölgeler veya tam tersi (nükleofilik) bölgeler tespit edilebilmektedir [38-39]. Kisaca molekülün fizikokimyasal özelliklerinin belirlenmesinde çok faydalı olduğu bilinmektedir. Elektron yoğunluğunun az olduğu bölgelerde atom çekirdeğinin protonu itmesinden oluşan potansiyel (+) yüklü elektrostatik potansiyel olarak bilinir ve mavi renk ile gösterilmektedir. Elektron yoğunluğunun fazla olduğu bölgelerde protonun çekilmesiyle oluşan potansiyel (-) yüklü potansiyel olarak bilinir ve kırmızı renk ile gösterilmektedir. Moleküldeki atomların etkileşimleri veya diğer moleküllerle olan etkileşimlerini anlayabilmek için bu bölgelerin 
belirlenmesi önem arz etmektedir. HMX ve ondan türetilen moleküller için DFT/B3LYP düzeyinde 6-31G** temel settinde elde edilen $\mathrm{V}(\mathrm{r})=0,001$ au izodensite yüzeyinde eşlenen elektrostatik potansiyeller Şekil 5'de görülmektedir. Şekil 5 incelendiğinde, elektron yoğunluğunun fazla olduğu (kırmızı) bölgelerin oksijen $(\mathrm{O})$ atomları civarında ve az olduğu (mavi) bölgelerin hidrojen (H) atomları ve N-H civarında olduğu görülmektedir. Nitro $\left(\mathrm{NO}_{2}\right)$ grupları yerine amino $\left(\mathrm{NH}_{2}\right)$ ve nitrozo (NO) gruplarının gelmesi ile bir miktar yoğunluk azalmaktadır ve en düşük yoğunluk 3-(2) $\mathrm{NH}_{2}-2$ için tespit edilmiştir.

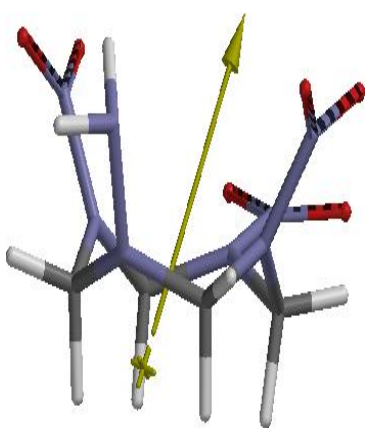

1-(1) $\mathrm{NH}_{2}$

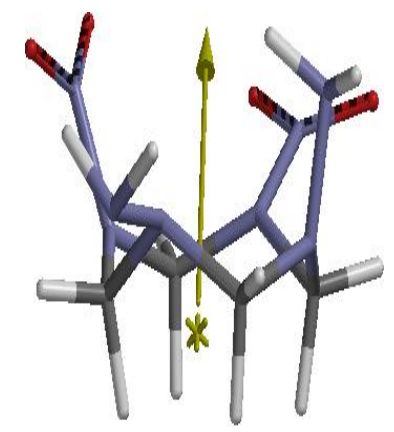

2-(2) $\mathrm{NH}_{2}-1$

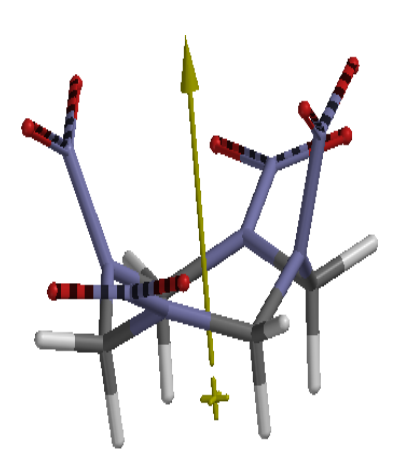

HMX

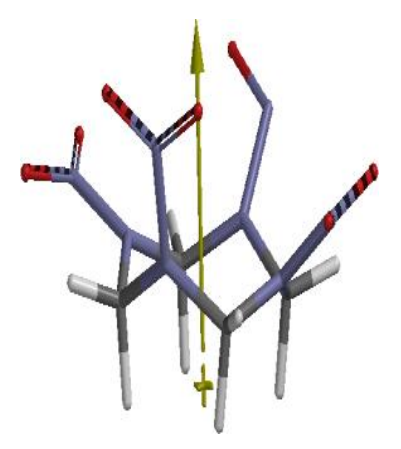

4-(1)NO

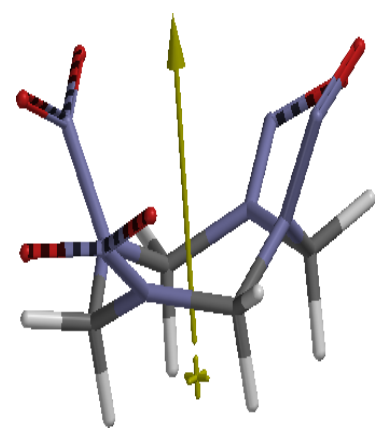

5-(2)NO-1

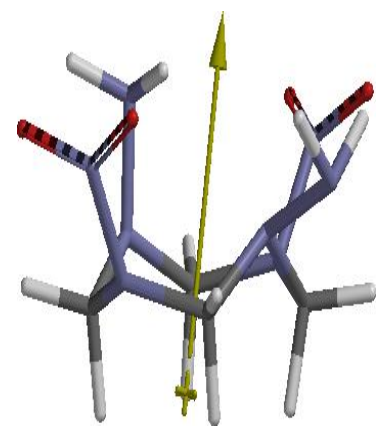

3-(2) $\mathrm{NH}_{2}-2$

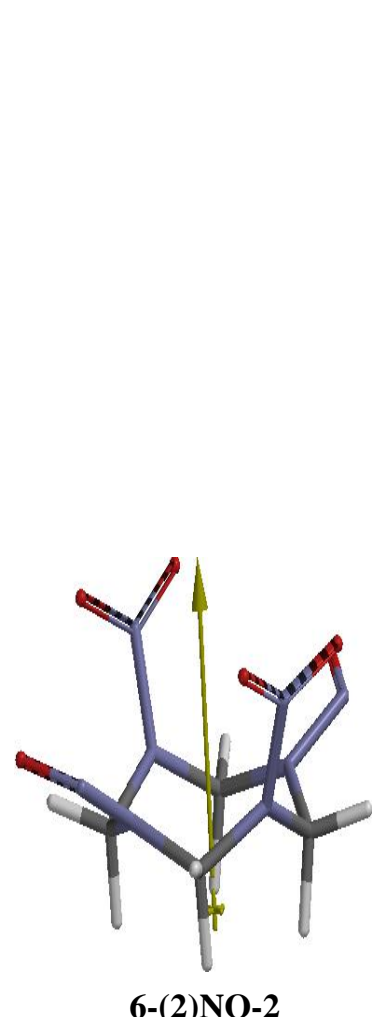

Şekil 4. HMX ve ondan türetilen moleküllerin optimize şekilleri 


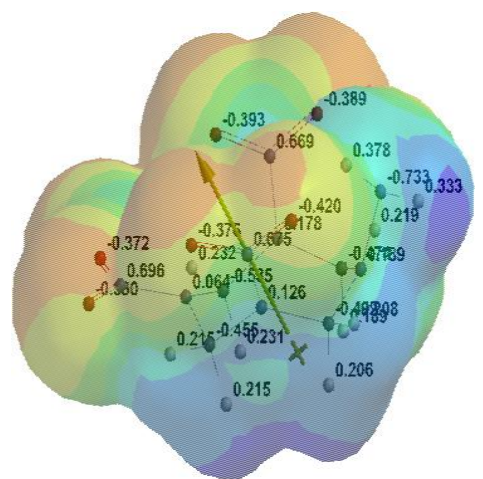

1-(1) $\mathrm{NH}_{2}$

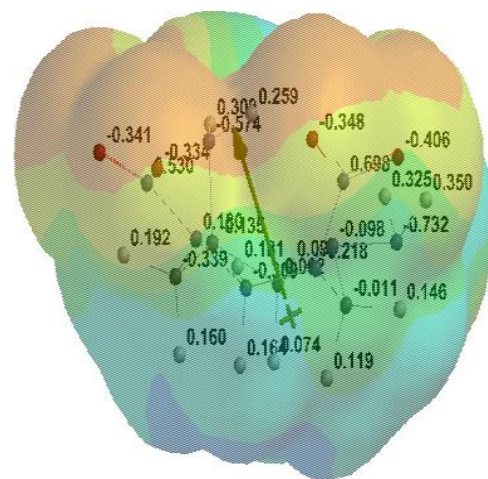

2-(2) $\mathrm{NH}_{2}-1$

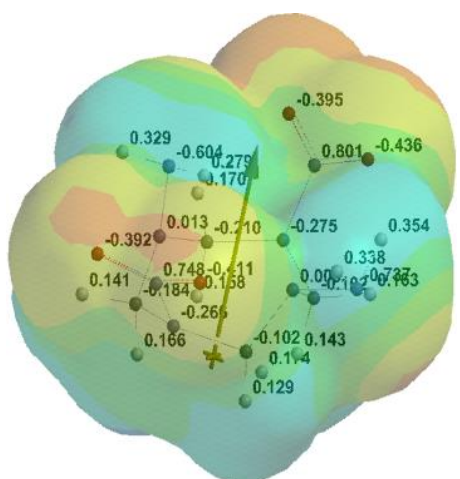

3-(2) $\mathrm{NH}_{2}-2$

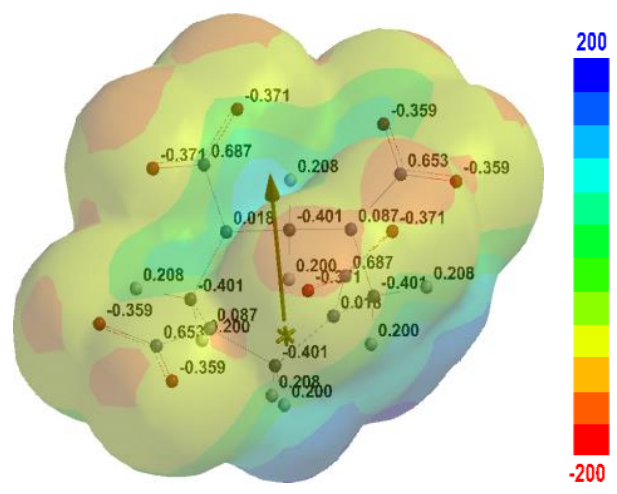

HMX

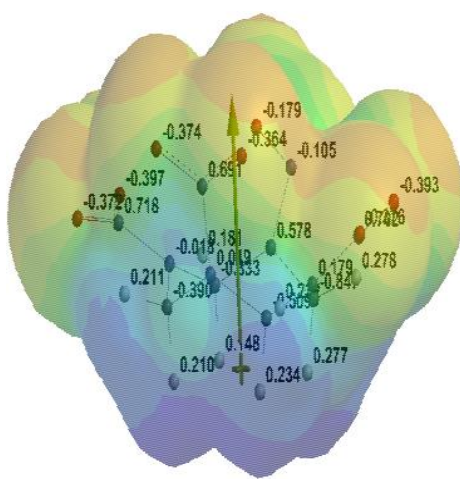

4-(1)NO

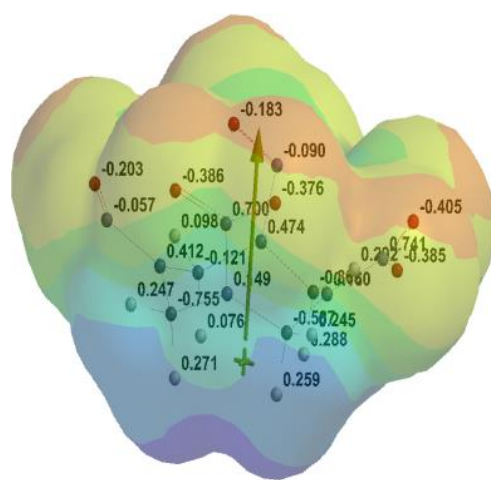

5-(2)NO-1

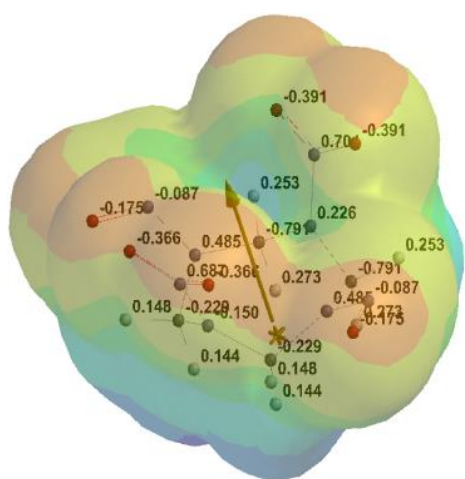

6-(2)NO-2

Şekil 5. HMX ve ondan türetilen moleküllerin $\mathrm{V}(\mathrm{r})=0,001$ au üzerinde eşlenen elektrostatik potansiyeller, -200 (kırmızı) ile +200 (mavi) arasında değişen izodensite yüzeyi 


\subsection{Balistik Özellikleri}

Patlayıcı maddeler, çarpma, şok, kıvılcım v.b. bir etkiye maruz kaldıklarında kendi kendine ilerleyen son derece hızlı ve karmaşık kimyasal reaksiyonlar oluştururlar. Sürecin hızlı olması nedeniyle yanma reaksiyonu için gerekli oksijen havadan sağlanamaz ve molekülün yapısındaki oksijenlerle tepkime devam eder [40]. Eşitlik 3 yardımıyla hesaplanan oksijen dengesi $(\% \Omega)$, molekülün oksitlenebilme ölçüsü olarak ifade edilebilir. Bir patlayıcı molekülü yapısındaki karbon (C), hidrojen (H), azot (N) ve yapisinda olan metallerden oksit üretebilecek oksijene sahip olması durumunda, sıfır oksijen dengesine sahiptir (Çizelge 3- EGDN). Molekülün ihtiyaç duyulandan fazla oksijene sahip olması durumunda pozitif oksijen dengesine (Çizelge 3- Nitrogliserin) ve tersinde ise negatif oksijen dengesine (Çizelge 3- Pikrik asit) sahip olması gerekmektedir. $\mathrm{Bu}$ çalışmadaki tüm moleküller, askeri çalışmalarda sıklıkla kullanılan TNT ve RDX gibi negatif oksijen dengesine sahiptir.

Çizelge 3. Bazı patlayıcı moleküllerinin, HMX ve onun türevlerinin oksijen değerleri

\begin{tabular}{|l|c|c|}
\hline Patlayıcı & Kimyasal formülü & \% \\
\hline Nitrogliserin & $\mathrm{C}_{3} \mathrm{H}_{5} \mathrm{~N}_{3} \mathrm{O}_{9}$ & 3,50 \\
\hline EGDN (Etilen glikol dinitrat) & $\mathrm{C}_{2} \mathrm{H}_{4} \mathrm{~N}_{2} \mathrm{O}_{6}$ & 0,00 \\
\hline PETN & $\mathrm{C}_{5} \mathrm{H}_{8} \mathrm{~N}_{4} \mathrm{O}_{12}$ & $-10,13$ \\
\hline RDX (Siklotrimetilen-trinitramin) & $\mathrm{C}_{3} \mathrm{H}_{6} \mathrm{~N}_{6} \mathrm{O}_{6}$ & $-21,60$ \\
\hline Pikrik asit (1-Hydroxy-2,4,6-trinitrobenzene) & $\mathrm{C}_{6} \mathrm{H}_{3} \mathrm{~N}_{3} \mathrm{O}_{7}$ & $-45,40$ \\
\hline HMX & $\mathrm{C}_{4} \mathrm{H}_{8} \mathrm{~N}_{8} \mathrm{O}_{8}$ & $-21,62$ \\
\hline 1 & $\mathrm{C}_{4} \mathrm{H}_{10} \mathrm{~N}_{8} \mathrm{O}_{6}$ & $-42,08$ \\
\hline 2 & $\mathrm{C}_{4} \mathrm{H}_{12} \mathrm{~N}_{8} \mathrm{O}_{4}$ & $-67,74$ \\
\hline 3 & $\mathrm{C}_{4} \mathrm{H}_{12} \mathrm{~N}_{8} \mathrm{O}_{4}$ & $-67,74$ \\
\hline 4 & $\mathrm{C}_{4} \mathrm{H}_{8} \mathrm{~N}_{8} \mathrm{O}_{7}$ & $-28,55$ \\
\hline 5 & $\mathrm{C}_{4} \mathrm{H}_{8} \mathrm{~N}_{8} \mathrm{O}_{6}$ & $-36,34$ \\
\hline 6 & $\mathrm{C}_{4} \mathrm{H}_{8} \mathrm{~N}_{8} \mathrm{O}_{6}$ & $-36,34$ \\
\hline TNT (Trinitrotoluen) & $\mathrm{C}_{7} \mathrm{H}_{5} \mathrm{~N}_{3} \mathrm{O}_{6}$ & $-73,98$ \\
\hline
\end{tabular}

Balistik özellikler, patlama basıncı ve patlama hızı olmak üzere iki önemli değişkenden oluşmaktadır. Patlayıcı bileşiklerin balistik niteliklerini etkileyen özelliklerden biri olan yoğunluk hesabı için Monte Carlo metodu, Eşitlik 1 ve 2'de verilen KamletJacobs denklemleri kullanılarak HMX molekülünün teorik olarak patlama hızı (D) ve patlama basıncı (P) hesaplanmış ve Çizelge 4'de verilmiştir. Çizelge 4'den görüleceği üzere bu çalıșmada elde edilen değerler ile literatür verileri uyum içerisindedir.

Çizelge 4. HMX molekülü için gaz fazında yoğunluk, patlama hızı ve patlama basıncı değerlerinin literatür verileri ve bu çalışmada elde edilen değerler

\begin{tabular}{|l|c|c|c|c|c|c|c|c|c|}
\hline & HMX & {$[\mathbf{1 3}]$} & {$[\mathbf{2 1}]$} & {$[\mathbf{4 1}]$} & {$[\mathbf{4 2}]$} & {$[\mathbf{4 3}]$} & {$[\mathbf{4 4}]$} & {$[\mathbf{4 5}]$} & {$[\mathbf{4 6}]$} \\
\hline $\mathbf{P}\left(\mathrm{g} / \mathrm{cm}^{3}\right)$ & 1,907 & 1,90 & 1,89 & 1,90 & 1,91 & 1,91 & 1,90 & 1,91 & 1,9 \\
\hline $\mathbf{D}(\mathrm{km} / \mathrm{s})$ & 9,24 & 9,10 & 9,03 & 9,10 & 9,20 & 9,25 & 8,89 & 9,32 & 9,14 \\
\hline $\mathbf{P}(\mathrm{GPa})$ & 39,19 & 39,30 & 39,00 & 39,00 & 38,90 & 39,31 & 36,2 & 39,6 & 39,5 \\
\hline
\end{tabular}


HMX ve ondan türetilen moleküllerin Kistiakowsky-Wilson kuralları [22] uygulanarak olası reaksiyon ürünleri aşağıdaki şekilde verilmiş olup patlama sonrası oluşabilecek toplam gaz miktarları Çizelge 5'de görülmektedir.
( $\mathrm{HMX)} \mathrm{C}_{4} \mathrm{H}_{8} \mathrm{~N}_{8} \mathrm{O}_{8} \rightarrow 4 \mathrm{CO}_{(\mathrm{g})}+4 \mathrm{H}_{2} \mathrm{O}_{(\mathrm{g})}+4 \mathrm{~N}_{2(\mathrm{~g})}$

(1) $\mathrm{C}_{4} \mathrm{H}_{10} \mathrm{~N}_{8} \mathrm{O}_{6} \rightarrow \mathrm{CO}_{(\mathrm{g})}+5 \mathrm{H}_{2} \mathrm{O}_{(\mathrm{g})}+4 \mathrm{~N}_{2}(\mathrm{~g})$

(2-3) $\quad \mathrm{C}_{4} \mathrm{H}_{12} \mathrm{~N}_{8} \mathrm{O}_{4} \rightarrow 4 \mathrm{H}_{2} \mathrm{O}_{(\mathrm{g})}+4 \mathrm{~N}_{2(\mathrm{~g})}+2 \mathrm{H}_{2}+4 \mathrm{C}_{(\mathrm{k})}$

(4) $\mathrm{C}_{4} \mathrm{H}_{8} \mathrm{~N}_{8} \mathrm{O}_{7} \rightarrow 3 \mathrm{CO}_{(\mathrm{g})}+4 \mathrm{H}_{2} \mathrm{O}_{(\mathrm{g})}+4 \mathrm{~N}_{2(\mathrm{~g})}+\mathrm{C}_{(\mathrm{k})}$

(5-6) $\quad \mathrm{C}_{4} \mathrm{H}_{8} \mathrm{~N}_{8} \mathrm{O}_{6} \rightarrow 2 \mathrm{CO}_{(\mathrm{g})}+4 \mathrm{H}_{2} \mathrm{O}_{(\mathrm{g})}+4 \mathrm{~N}_{2(\mathrm{~g})}+2 \mathrm{C}_{(\mathrm{k})}$

Çizelge 5. HMX ve ondan türetilen moleküllerin patlama sonrası oluşabilecek olası gaz molekülleri.

\begin{tabular}{|c|c|c|c|c|c|c|c|}
\hline & Formül & $\mathbf{N}_{2}$ & $\mathbf{H}_{2} \mathbf{O}$ & $\mathbf{C O}$ & $\mathbf{H}_{2}$ & $\mathbf{C}_{(\mathbf{k})}$ & $\mathbf{N}_{\text {Toplam gaz }}$ \\
\hline $\mathrm{HMX}$ & $\mathrm{C}_{4} \mathrm{H}_{8} \mathrm{~N}_{8} \mathrm{O}_{8}$ & 4 & 4 & 4 & - & - & 12 \\
\hline 1 & $\mathrm{C}_{4} \mathrm{H}_{10} \mathrm{~N}_{8} \mathrm{O}_{6}$ & 4 & 5 & 1 & - & 3 & 10 \\
\hline 2 & $\mathrm{C}_{4} \mathrm{H}_{12} \mathrm{~N}_{8} \mathrm{O}_{4}$ & 4 & 4 & - & 2 & 4 & 10 \\
\hline 3 & $\mathrm{C}_{4} \mathrm{H}_{12} \mathrm{~N}_{8} \mathrm{O}_{4}$ & 4 & 4 & - & 2 & 4 & 10 \\
\hline 6 & $\mathrm{C}_{4} \mathrm{H}_{8} \mathrm{~N}_{8} \mathrm{O}_{7}$ & 4 & 4 & 3 & - & 1 & 11 \\
\hline 7 & $\mathrm{C}_{4} \mathrm{H}_{8} \mathrm{~N}_{8} \mathrm{O}_{6}$ & 4 & 4 & 2 & - & 2 & 10 \\
\hline 8 & $\mathrm{C}_{4} \mathrm{H}_{8} \mathrm{~N}_{8} \mathrm{O}_{6}$ & 4 & 4 & 2 & - & 2 & 10 \\
\hline
\end{tabular}

Çizelge 6' da HMX ve ondan türetilen moleküllerin B3LYP düzeyinde 6-31G** temel setinde elde edilen ve hesaplamalar yapilarak bulunan balistik özelliklerle ilgili TNT $<$ PA $<2<3<1<6<5<$ $4<$ HMX şeklinde bir performans siralaması yapılabilir. Nitro $\left(\mathrm{NO}_{2}\right)$ gruplarının nitrozo (NO) grupları ve amino $\left(\mathrm{NH}_{2}\right)$ grupları ile yer değiştirmesi balistik özellikleri azaltırken, amino gruplarındaki düşüş nirtozo gruplarına göre daha fazladır. Yoğunluklar bakımından değerlendirme yapılacak olursa yine aynı şekilde amino gruplarının nitrozo gruplarına göre daha düşük yoğunluğa sahip olduğu görülmektedir. Çizelge 6'da verilen oluşum 1sısı $\left(\Delta \mathrm{H}_{\mathrm{f}}^{\mathrm{o}}\right)$ değerleri Parametrizasyon metodu (PM3) T1 enerji düzeyinde kullanılarak gaz fazında hesaplanmıştır [15]. Patlama basınc1 ve patlama hizı her bir molekül için patlama tepkimelerinin oksijen dengesi $(\Omega)$ kurallarına uydukları varsayılarak hesaplanmıştır.

Çizelge 6. HMX ve ondan türetilen moleküller için elde edilen teorik sonuçlar.

\begin{tabular}{|c|c|c|c|c|c|c|c|c|c|}
\hline & $\mathbf{\rho}$ & $\% \mathbf{\Omega}$ & $\mathbf{V}$ & $\mathbf{N}$ & $\mathbf{M}$ & $\mathbf{Q}$ & $\Delta \mathbf{H}_{\mathbf{f}}^{\mathbf{1}}$ & $\mathbf{D}$ & $\mathbf{P}$ \\
\hline & $\left(\mathrm{g} / \mathrm{cm}^{3}\right)$ & & $\left(\mathrm{cm}^{3} / \mathrm{mol}\right)$ & $(\mathrm{mmol} / \mathrm{g})$ & $(\mathrm{g} / \mathrm{mol})$ & $(\mathrm{kJ} / \mathrm{g})$ & $(\mathrm{kJ} / \mathrm{mol})$ & $(\mathrm{km} / \mathrm{s})$ & $(\mathrm{GPa})$ \\
\hline HMX & 1,907 & $-21,61$ & 155,29 & 33,77 & 27,20 & 1542,05 & 156,43 & 9,24 & 39,19 \\
\hline 1 & 1,813 & $-42,08$ & 146,82 & 35,69 & 23,58 & 1439,87 & 197,62 & 8,69 & 33,68 \\
\hline 2 & 1,687 & $-67,74$ & 140,09 & 38,11 & 19,56 & 1311,26 & 238,32 & 7,97 & 27,06 \\
\hline 3 & 1,699 & $-67,74$ & 139,03 & 38,10 & 19,56 & 1313,57 & 240,61 & 8,01 & 27,47 \\
\hline 4 & 1,889 & $-28,55$ & 148,30 & 33,91 & 26,32 & 1509,29 & 211,56 & 9,07 & 37,57 \\
\hline 5 & 1,888 & $-36,34$ & 139,94 & 34,10 & 25,33 & 1472,30 & 266,43 & 8,95 & 36,53 \\
\hline 6 & 1,873 & $-36,34$ & 141,02 & 34,11 & 25,33 & 1473,80 & 268,10 & 8,90 & 35,99 \\
\hline
\end{tabular}

*[47]

Amino grubu değişimlerinin (Çizelge 6-1, 2 ve 3) ve nitrozo grubu değişimlerinin (Çizelge 6- 4, 5 ve 6) duyarlılığı azaltmada etkili oldukları görülmektedir. Ancak amino grubu değişimleri, balistik özelliklerde ana yapıya göre çok fazla değişikliğe sebep olduğu için duyarlılığı azaltmada nitrozo grubu değişimleri daha etkin bir yoldur.
Patlama reaksiyonu sonunda ortama sıcak gaz ve ıs1 verildiği bilinmektedir. Açı̆̆a çıkan gazın hacmi patlayıcı molekülün yapmış olduğu iş ile ilgili bilgi verebilir [48-52]. Bu nedenle oluşan gazın hacmini belirlemek için standart koşullarda ( $273 \mathrm{~K}, 1 \mathrm{~atm}, 22,4 \mathrm{~L})$ hesaplama yapmak gerekir. Patlamada oluşan toplam gaz hacminin molekül ağırlığına bölünmesiyle bir gram patlayıcı 
molekülü başına ne kadar gaz açığa çıkacağı bulunabilir.

Patlayıcının gücü, patlama 1sısı (Q) ve oluşan gazın hacminin (v) çarpımı olarak gösterilebilir [22].

Patlayıcı Gücü= Q.v

Eşitlik 5 yardımıyla, her bir molekül için hesaplanan güç değerleri, Pikrik Asitin (PA) güç değeri ile kıyaslanarak güç endeksi verileri elde edilebilir (Çizelge 7).
$\%$ Güç Endeksi $=\frac{\mathrm{Q} \cdot \mathrm{v}}{\mathrm{QPA} \cdot \mathrm{vPA}_{\mathrm{PA}}} \times 100$

Çizelge 7'de görüleceği üzere HMX ve ondan türetilen moleküllerin güç endeksi değerleri $\%$ 106,09 ile 118,86 arasındadır ve HMX > $4>6>5$ $>3>2>1>\mathrm{PA}>\mathrm{TNT}$ şeklinde sıralanabilir. HMX'ten türetilen tüm yapıların, geniş çapta kullanılan PA ve TNT gibi patlayıcılardan daha yüksek güç endeksine sahip oldukları görülmektedir.

Çizelge 7. Pikrik asit, TNT, RDX, HMX ve ondan türetilen moleküllerin güç endeksi verileri

\begin{tabular}{|c|c|c|c|c|}
\hline Bileşik & $\mathbf{Q}\left(\mathrm{kJ} \cdot \mathrm{g}^{-1}\right)$ & $\boldsymbol{v}\left(\mathrm{dm}^{3} \cdot \mathrm{g}^{-1}\right)$ & $\mathbf{Q}^{*} \mathbf{v}$ & \% Güç Endeksi \\
\hline HMX & 1542,05 & 0,908 & 1400,18 & 118,86 \\
\hline 1 & 1439,87 & 0,842 & 1212,37 & 102,92 \\
\hline 2 & 1311,26 & 0,949 & 1244,39 & 105,63 \\
\hline 3 & 1313,57 & 0,949 & 1246,58 & 105,82 \\
\hline 4 & 1509,29 & 0,879 & 1326,67 & 112,62 \\
\hline 5 & 1472,30 & 0,848 & 1248,51 & 105,98 \\
\hline 6 & 1473,80 & 0,848 & 1249,78 & 106,09 \\
\hline PA (Pikrik Asit) & 1417,61 & 0,831 & 1178,03 & 100,00 \\
\hline TNT(Trinitrotoluen) & 1424,11 & 0,740 & 1053,35 & 89 \\
\hline
\end{tabular}

\section{SONUÇLAR VE ÖNERILLER}

HMX molekülünün DFT/B3LYP düzeyinde 6$31 \mathrm{G}^{* *}$ temel setinde optimizasyonu yapılarak belirlenmiş olan bağ uzunluğu ve bă̆ açıları verilerinin literatür değerleriyle uyum içerisinde olduğu görülmektedir. HMX'in nitro $\left(\mathrm{NO}_{2}\right)$ gruplarının nitrozo (NO) grupları ve amino $\left(\mathrm{NH}_{2}\right)$ gruplarına dönüştürülmesiyle bir patlayıcının duyarlılığını önemli bir güç kaybı olmadan, özellikle nitrozo gruplarının azaltma olasılığ kanıtlanmıştır. Türetilen bütün moleküllerin, TNT (Trinitrotoluen) ve PA'dan (Pikrik asit) daha iyi birer patlayıcı oldukları görülmektedir. Nitro $\left(\mathrm{NO}_{2}\right)$ gruplarının amino $\left(\mathrm{NH}_{2}\right)$ gruplarına dönüştürülmesi, nitrozo (NO) dönüşümlerine kıyasla daha duyarsız patlayıcılar oluşturmaktadır. Düşük hassasiyetli uygulamalar gerektiğinde, türetilen moleküllerin herhangi biri kullanılabilir. $\mathrm{Bu}$ çalışma, moleküler modifikasyonun HMX'in duyarsızlaştırılmasında etkili bir yöntem olduğunu göstermiştir.

\section{KAYNAKLAR}

1. Mamand, D.M., 2019. Theoretical Calculations and Spectroscopic Analysis of Gaussian Computational Examination-NMR, FTIR, UVVisible, MEP on 2,4,6-Nitrophenol. Journal of Physical Chemistry and Functional Materials, 2(2), 77-86.

2. Priya, M.K., Revathi, B.K., Renuka, V., Sathya, S., Asirvatham, P.S., 2019. Molecular Structure, Spectroscopic (FT-IR, FT-Raman, 13C and 1H NMR) Analysis, HOMO-LUMO Energies, Mulliken, MEP and Thermal Properties of New Chalcone Derivative by DFT Calculation. Materials Today: Proceedings, 8, 37-46.

3. Al-Ahmary, K.M., Habeeb, M.M., Aljahdali, S.H., 2019. Synthesis, Spectroscopic Studies and DFT/TD-DFT/PCM Calculations of Molecular Structure, Spectroscopic Characterization and NBO of Charge Transfer Complex Between 5-amino-1,3- 
dimethylpyrazole (5-ADMP) with Chloranilic Acid (CLA) in Different Solvents. Journal of Molecular Liquids, 277, 453-470.

4. Wu, X., Liu, Z., Ji, G., Zhu, W., 2019. Pressure-induced Structure, Vibrational Properties, and Initial Decomposition Mechanisms of Delta-HMX Crystal: A Periodic DFT Study. Journal of Molecular Graphics and Modelling, 90, 144-152.

5. Bari, R., Koh, Y.P., McKenna, G.B., Simon, S.L., 2020. Decomposition of HMX in Solid and Liquid States Under Nanoconfinement. Thermochimica Acta, 686.

6. Bondarchuk, S.V., 2020. Modeling of Explosives: 1,4,2,3,5,6-dioxatetrazinane as a New Green Energetic Material with Enhanced Performance. Journal of Physics and Chemistry of Solids, 142.

7. Zeng, Y., Song, Y., Yu, G., Zheng, X., 2016. A Comparative Study of 1,3,5-Trinitroperhydro1,3,5-triazine (RDX) and Octahydro-1,3,5,7tetranitro-1,3,5,7-tetrazocine (HMX) Under High Pressures Using Raman Spectroscopy and DFT Calculations. Journal of Molecular Structure, 1119, 240-249.

8. Jeong, K., Sung, I., Uk Joo, H., Kwon, T., Yuk, J.M., Kwon, Y., Kim, H., 2020. Molecular Design of Nitro-oxide-substituted Cycloalkane Derivatives for High-energy-density Materials. Journal of Molecular Structure, 1212.

9. Wang, P., Wang, J., Wang, J., 2020. Crystal Structure and Thermal Decomposition Kinetics of 1,3,5-trinitro-4,6-diazidobenzene. Journal of Thermal Analysis and Calorimetry, 143(6), 3983-3995.

10. Türker, L., 2019. Epoxides of DADNE Isomers-A DFT Study. Earthline Journal of Chemical Sciences, 121-139.

11. Shi, Y.B., Bai, L.F., Li, J.H., Sun, G.A., Gong, J., Ju, X., 2019. Theoretical Calculation into the Effect of Molar Ratio on the Structures, Stability, Mechanical Properties and Detonation Performance of 1,3,5,7-tetranitro1,3,5,7-tetrazocane/ 1,3,5-trinitro-1,3,5triazacyco-hexane Cocrystal. Journal of Molecular Modeling, 25(9), 299.

12. Shi, Y., Bai, L., Gong, J., Ju, X., 2019. Theoretical Calculation into the Structures, Stability, Sensitivity, and Mechanical
Properties of 2,4,6,8,10,12-hexanitro2,4,6,8,10,12 hexaazai-sowurtzitane (CL-20)/1amino-3-methyl-1,2,3-triazoliumnitrate (1AMTN) coCRYSTAL and its Mixture. Structural Chemistry, 31(2), 647-655.

13. Lin, H., Zhu, Q., Huang, C., Yang, D.D., Lou, N., Zhu, S.G., Li, H.Z., 2019. Dinitromethyl, Fluorodinitromethyl Derivatives of RDX and HMX as High Energy Density Materials: a Computational Study. Structural Chemistry, 30(6), 2401-2408.

14. Lin, H., Yang, D.D., Lou, N., Zhu, S.G., Li, H.Z., 2019. Theoretical Design and Screening Potential High Energy Density Materials: Combination of 1,2,4-oxadiazole and 1,3,4oxadiazole Rings. Combustion, Explosion, and Shock Waves, 55(5), 547-554.

15. Türker, L., Variş, S., 2017. Structurally Modified RDX-A DFT Study. Defence Technology, 13(6), 385-391.

16. Spartan, 2005. Molecular Modeling in Physical Chemistry, 52-57.

17. Frisch, M.J., Trucks, G., Schlegel, H.B., Scuseria, G.E., Robb, M., Cheeseman, J., Jr, J.A., Vreven, T., Kudin, K.N., Burant, J.C., 2004. Gaussian 03, Revision D.01. Gaussian, Inc., Wallingford, CT.

18. Türker, L., 2019. Interaction of TATB with $\mathrm{Cu}$ and $\mathrm{Cu}+1$. A DFT Study, Defence Technology, 15(1), 27-37.

19. Young, D.C., 2001. Computational Chemistry: A Practical Guide for Applying Techniques to Real-World Problems. John Wiley \& Sons, Inc,

20. Türker, L., Variş, S., 2013. Prediction of Explosive Performance Properties ofz-DBBD and its Isomers by Quantum Chemical Computations. Journal of Energetic Materials, 31(3), 203-216.

21. Wu, Q., Yan, G., Li, M., Hu, Q., Zhang, Z., Zhu, W., 2020. Density Functional Theory Studies of Effects of Boron Replacement on the Structure and Property of RDX and HMX. Journal of the Chinese Chemical Society, 67(11), 1977-1985.

22. Akhavan, J., 1998. The Chemistry of Explosives United Kingdom: RSC Paperback 37-38, 73-74.

23. Yang, J., Wang, F., Zhang, J., Wang, G., Gong, X., 2013. A Theoretical Study on 1,5-diazido- 
3-nitrazapentane (DANP) and 1,7-diazido2,4,6-trinitrazaheptane (DATNH): Molecular and Crystal Structures, Thermodynamic and Detonation Properties, and Pyrolysis Mechanism. Journal of Molecular Modeling, 19(12), 5367-76.

24. Pu, K., Wang, L., Liu, J., Zhong, K., 2020. Theoretical Design of Bis-azole Derivatives for Energetic Compounds. RSC Advances, 10(22), 13185-13195.

25. Wang, G., Xu, Y., Zhang, W., Gong, X., 2019. A Theoretical Study of Polyethylene Glycol Polynitrates as Potential Highly Energetic Plasticizers for Propellants. Central European Journal of Energetic Materials, 16(2), 194-215.

26. Qiu, L., Xiao, H., Gong, X., Ju, X., Zhu, W., 2006. Theoretical Studies on the Structures, Thermodynamic Properties, Detonation Properties, and Pyrolysis Mechanisms of Spiro Nitramines. The Journal of Physical Chemistry A, 110, 3797-3807.

27. Qiu, L., Xiao, H., Gong, X., Ju, X., Zhu, W., 2006. Theoretical Studies on the Structures, Thermodynamic Properties, Detonation Properties, and Pyrolysis Mechanisms of Spiro Nitramines. Journal of Computational Chemistry A, 110(10), 3797-3807.

28. Kamlet, M.J., Jacobs, S.J., 1968. Chemistry of Detonations. I. A Simple Method for Calculating Detonation Properties of $\mathrm{C} \mathrm{H} \mathrm{N} \mathrm{O}$ Explosives. The Journal of Chemical Physics, 48, 23-25

29. Kamlet, M.J., Short, J.M., 1980. The Chemistry of Detonations. VI. A "Rule for Gamma" as a Criterion for Choice Among Conflicting Detonation Pressure Measurements. Combustion and Flame, 38, 221-230.

30. Jeong, K., 2018. New Theoretically Predicted RDX- and $\beta$-HMX-based High-energy-density Molecules. International Journal of Quantum Chemistry, 118(6), 1-7.

31. Choi, C.S., Boutin, H.P., 1970. A Study of the Crystal Structure of $[\beta]$-cyclotetramethylene Tetranitramine by Neutron Diffraction. Acta Crystallographica, B26, 1235-1240.

32. Lewis, J.P., 2003. Energetics of Intermolecular HONO Formation in Condensed-phase octahydro-1,3,5,7-tetranitro-1,3,5,7-tetrazocine
(HMX). Chemical Physics Letters, 371(5-6), 588-593.

33. Damian, G.A., Darya, A.P., Timothy, M.K., 2006. Solid-State Modeling of the Terahertz Spectrum of the High Explosive HMX. Journal of Physical Chemistry A 110, 1951-1959.

34. Palmer, S.J.P., Fieldf, J.E.,F., 1982. The Deformation and Fracture of $(\beta-H M X)$. Proceedings of the Royal Society of London, A 383, 399-407.

35.Lu,L.Y., Wei, D.Q., Chen, X.R., Ji, G.F., Wang, X.J., Chang, J., Zhang, Q.M., Gong, Z.Z., 2009. The Pressure-induced Phase Transition of the Solid $\beta$-HMX. Molecular Physics, 107(22), 2373-2385.

36. Shaowen, Z., Thanh, N.T., 2000. Thermal Rate Constants of the NO2 Fission Reaction of Gas Phase $\beta$-HMX: A Direct ab Initio Dynamics Study. Journal of Physical Chemistry A 104, 7304-7307.

37. Lewis, J.P., Glaesemann, K.R., VanOpdorp, K., Voth, G.A., 2000. Ab Initio Calculations of Reactive Pathways for r-Octahydro-1,3,5,7tetranitro-1,3,5,7-tetrazocine $(\beta$-HMX). J. Phys. Chem. A, 104, 11384-11389.

38. Gümüş, H., 2019. Nikotinamid İçeren Çinko (II) Kompleksinin Moleküler Özellikleri ve Titreşim Spektrumu. Gümüşhane Üniversitesi Fen Bilimleri Enstitüsü Dergisi, 9(2), 254-261.

39. Göcen T., Güven M.H., 2020. Palmitoleik Asidin Moleküler Yapısı, Titreşim Spektrumları ve Elektronik Özelliklerinin Teorik Olarak İncelenmesi. Bilecik Şeyh Edebali Üniversitesi, Fen Bilimleri Dergisi, 7(2), 553-573.

40. Bozkuş, S.İ., Şen, N., 2019. 2,4,6-Trinitrofenol ve Trinitrotoluen ile 1-Bromopiren Arasında Oluşan Yeni Enerjik Ko-kristalin Karakterizasyonu ve Enerjik Performans Özellikleri. Süleyman Demirel Üniversitesi, Fen Edebiyat Fakültesi, Fen Dergisi, 14, 136-149.

41. Talawar, M.B., Sivabalan, R., Mukundan, T., Muthurajan, H., Sikder, A.K., Gandhe B.R., Rao, S., 2009. Environmentally Compatible Next Generation Green Energetic Materials (GEMs). Journal of Hazardous Materials, 161(2-3), 589-607. 
42. Pan, Y., Zhu, W., Xiao, H., 2013. DFT Studies on Trinitromethyl- or Dinitromethyl-modified Derivatives of RDX and $\beta$-HMX, Computational and Theoretical Chemistry, 1019, 116-124.

43. Jeon, W.C., Lee, H.Y., Kim, J.C., Kang, S.J., jung, S.H., Cho, S.G., Kwak, S.K., 2020. Reaction Kinetics of Mixture of Nitromethane and Detonator Confined in Carbon Nanotube. Journal of Industrial and Engineering Chemistry, 83, 64-71.

44. Shang, Y., Huang, R.K., Chen, S.L., He, C.T., Yu, Z.H., Ye, Z.M., Zhang, W.X., Chen, X.M., 2020. Metal-Free Molecular Perovskite HighEnergetic Materials. Crystal Growth \& Design, 20(3), 1891-1897.

45. Zhang, Y., Li, Y., Hu, J., Ge, Z., Sun, C., Pang, S., 2019. Energetic C-trinitromethyl-substituted Pyrazoles: Synthesis and Characterization. Dalton Transactions, 48(4), 1524-1529.

46. Liu, J., Liu, L., Liu, X., 2019. Development of High-energy-density Materials. Science China Technological Sciences, 63(2), 195-213.

47. Wang, G., Xiao, H., Ju, X., Gong, X., 2006. Calculation of Detonation Velocity, Pressure, and Electric Sensitivity of Nitro Arenes Based on Quantum Chemistry. Propellants, Explosives, Pyrotechnics, 31(5), 361-368.

48. Xu, W., Guo, F., Liang, X., Yan, T., Xu, Y., Deng, J., Li, Y., Wang, J., 2021. Dynamic Response Properties of Polymer Bonded Explosives Under Different Excitation by Deceleration. Materials \& Design, 206, 109810-109825.

49. Perry, W. L., Duque, A. L., Mang, J. T., Culp, D.B., 2021. Computing Continuum-level Explosive Shock and Detonation Response Over a Wide Pressure Range from Microstructural Details. Combustion and Flame, 231, 111470-111483.

50. Liu, D., Zhao, P., Chan, S.H., Hng, H.H., Chen, L., 2021. Effects of Nano-sized Aluminum on Detonation Characteristics and Metal Acceleration for RDX-based Aluminized Explosive. Defence Technology, 17(2), 327-337.

51. Bondarchuk, S.V., 2021. Diazoamination: A Simple way to Enhance Detonation Performance of Aminoaromatic and
Aminoheterocyclic Energetic Materials. Fire Phys Chem, 1(2), 97-102.

52. Choi, S., Kim, B., Han, S., Yoh, J.J., 2020. Multiscale Modeling of Transients in the Shock-induced Detonation of Heterogeneous Energetic Solid Fuels. Combustion and Flame, 221, 401-415. 
